\title{
The Effect of Ultra Fine Bubbles Water on the Human skin
}

\author{
Yuji Kato $^{\mathrm{a}}$, Tomoyuki Matsumoto ${ }^{\mathrm{a}}$, and Setsuko Koura ${ }^{\mathrm{a}}$ \\ ${ }^{a}$ Chiba Institute of Technology \\ Narashino-city, Chiba 275-0016, Japan
}

\begin{abstract}
A certain amount of water needs to be maintained in the stratum corneum of the skin in order to maintain the skin barrier function. Therefore, it is important to supply water to the stratum corneum of the skin to reduce trans epidermal water loss (TEWL). However, because normal water has large clusters, it is difficult to penetrate the stratum corneum of the skin. Therefore, it was considered that the use of Ultra-fine bubbles (UFB) water, which is said to have small water clusters, promotes penetration into the stratum corneum of the skin, and is useful for improving the skin barrier function. The artificial skin to which $\mathrm{O}_{2}$-UFB water was dripped had the highest water content and the lowest TEWL. It also had a high affinity for human skin. From these results, improvement of skin barrier function by $\mathrm{O}_{2}$-UFB water can be expected.
\end{abstract}

\section{Introduction}

Skin barrier function refers to a role of preventing evaporation of moisture from the body and a role of protecting the body from invasion and irritation of foreign matter from the outside. In order to maintain the skin barrier function is a physiological function of the skin, a certain amount of water must be retained in the stratum corneum of the skin, so it is necessary to supply water to the stratum corneum of the skin and prevent trans epidermal water loss (TEWL) ${ }^{1)}$. Lotions and moisturizers are commonly used as countermeasures ${ }^{2)}$. However, the preservatives and surfactants contained therein have an adverse effect on children with weak skin, elderly people, patients with atopic predisposition, etc., such as inducing skin dryness and inflammation ${ }^{3)}$. Therefore, methods for improving the skin barrier function that have little effect on the human body are being studied.

So we focused on Ultra-fine bubbles (UFB). Bubbles are familiar to us, but when we put these bubbles into a fine state, they have different characteristics than ordinary bubbles. UFB refers to bubbles having a diameter of $1 \mu \mathrm{L}$ or less, has a lower floating speed in water than ordinary bubbles, with unique features that has a property of target gas in water 
for a long time ${ }^{4)}$. Studies have been conducted in various fields, and it has been reported that the agricultural field has a growth promoting effects on plants and the fishing field has a fish growth promoting effect and the removal of norovirus from oysters. It is also said that bubbling at the time of UFB water production subdivide water clusters and lowers surface tension ${ }^{5)}$. Since ordinary water has a large cluster, it is difficult to penetrate into the stratum corneum of the skin. Therefore, we thought that the use of UFB water with small water clusters would promote penetration of the skin into the stratum corneum and help improve the function of the body-friendly skin barrier.

In this paper, we evaluated the effects of UFB water on the human skin.

\section{Experimental}

\section{Generation of oxygen UFB water}

UFB water was prepared by gas-liquid mixed shear method with compact generator (Sunstar Inc.). In this equipment, UFB water was produced under the conditions of gas flow rate of $0.45 \mathrm{~L} / \mathrm{min}$, number of revolutions $4000 \mathrm{rpm}$, operating time $10 \mathrm{~min}$, and amount of pure water $1 \mathrm{~L}$. The kinds of gas selected were oxygen, air, nitrogen and argon (TABLE 1).

The dissolved oxygen (DO) concentration in the water was measured by DO meter (Thermo Fisher Scientific Co). The meter can measure the DO concentration 0 to $50 \mathrm{mg} / \mathrm{L}$. Size distribution of oxygen UFB was measured by laser scattering Nano-Sight LM10HFV (Malvarn Panalytical).

TABLE 1. UFB Water Production Conditions.

\begin{tabular}{cc}
\hline Tap Water & $1.0 \mathrm{~L}$ \\
Gas Species & $\mathrm{O}_{2}, \mathrm{~N}_{2}, \mathrm{Ar}, \mathrm{Air}$ \\
(purity $99.99 \%)$ \\
Gas Flow Rate & $0.45 \mathrm{~L} / \mathrm{min}$ \\
Time & $10.0 \mathrm{~min}$ \\
Rotation Speed & $4000 \mathrm{rpm}$ \\
\hline
\end{tabular}

\section{Preparation mechanism of UFB}

Figure 1 show a schematic diagram of the UFB by a gas-liquid mixing shear method. The microbubble generated by mixing the gas and liquid from the IN side is sent to the UFB generator at the pressure of the pump. The microbubbles are sheared by centrifugal force to generate the UFB. 


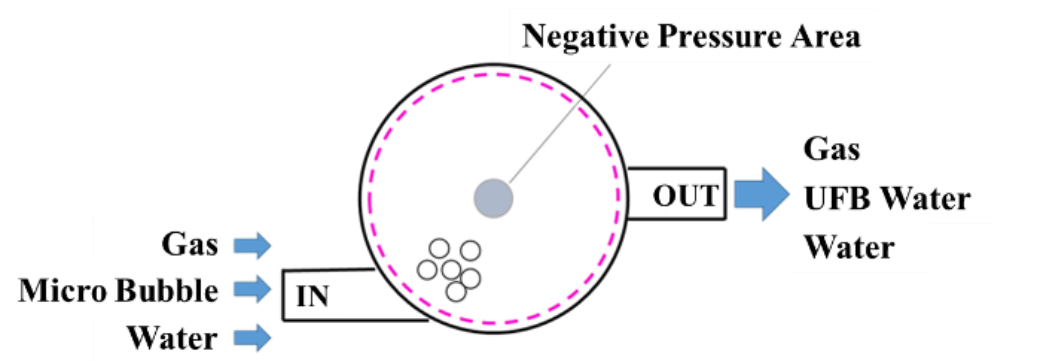

Source) Oxygen and Air Nanobubble Water Solution Promote the Growth of Plants, Fishes, and Mice

Figure 1. Generation diagram of UFB by gas-liquid mixing shear method

\section{Evaluation of the effect of UFB water on skin model}

The skin model used was Human three-dimensional cultured epidermis LabCyte EPI-MODEL24 (Japan Tissue Engineering Co., Ltd.) in which human normal epidermal cells were cultured in multiple layers (Figure 2).

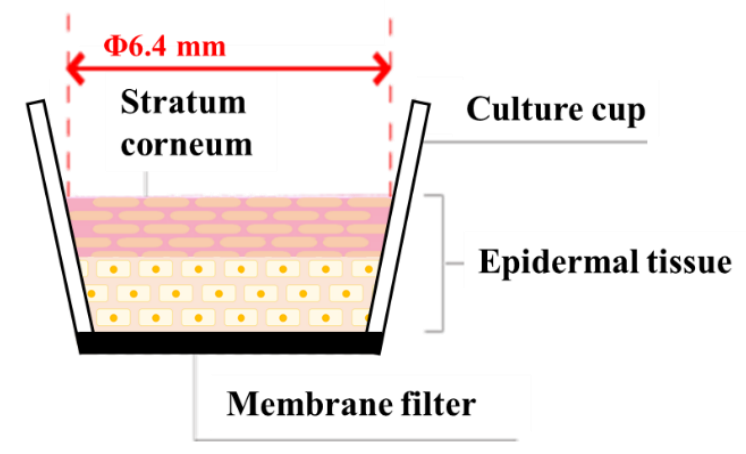

Figure 2. Schematic diagram of skin model

$50 \mu \mathrm{L}$ each of pure water and UFB water containing each gas were added dropwise to the skin model. Then, it was dried, and the measurement and the influence of each solution were compared. The measurement was carried out in a constant environment where the room temperature $22 \pm 1{ }^{\circ} \mathrm{C}$ and the relative humidity was $60 \%$.

In the irritating test, the viable cell rate in the MTT reaction was measured.

The amount of water in the stratum corneum was measured by using the stratum corneum film thickness and moisture meter ASA-MX100 (ASCH JAPAN Co., LTD).

TEWL, using the transdermal moisture transpiration measuring device VAPO SCAN AS-VT100RS (ASCH JAPAN Co., LTD), by the closed chamber humidity change measurement method, was measured. 


\section{Evaluation of contact angle of UFB water to the human skin}

Tape stripping samples were taken from the skin inside the forearm of the subject (author: male in his 20s). The environment at the time of collection was carried out in a constant environment where the room temperature $22 \pm 1{ }^{\circ} \mathrm{C}$ and the relative humidity was $60 \%$.

Cellophane tape (Nichiban Co., Ltd.) cut into $15 \times 40 \mathrm{~mm}$ was placed on the skin and pressed with a silicone rubber plate of $30 \times 50 \times 8 \mathrm{~mm}$ under a load of $400 \mathrm{~g} / \mathrm{cm}^{2}$ for 3 seconds. Then, the tape was peeled off to collect the stratum corneum. Next, using the stratum corneum collected by tape stripping and cellophane tape that has not been operated as a comparison target (control) as a substrate, $1 \mu \mathrm{L}$ of pure water and UFB water containing each gas was dropped on each. Thereafter, the contact angle of the generated droplets was measured using a contact angle meter DM300 (Kyowa Interface Science Co., Ltd.) to compare the affinity of each solution with the skin.

\section{Results and Discussion}

\section{Safety of UFB to the skin}

The results when measuring the viable cell rate in the MTT reaction are show in the Table 2. Since the viable cell rate of SLS added dropwise as a positive control was $40 \%$ or less, it can be said that the test could be performed normally. Moreover, the viable cell rate of UFB was $85 \%$ or more in all gas species. Therefore, it was suggested that UFB water has no irritation to the skin.

TABLE 2. Viable Cell Rate in Each Samples.

\begin{tabular}{cc}
\hline Sample Name & Viable Cell Rate (\%) \\
\hline Pure Water (Negative Control) & 100 \\
SLS (Positive Control) & 14.6 \\
$\mathrm{O}_{2}$-UFB Water & 85.9 \\
$\mathrm{~N}_{2}$-UFB Water & 93.1 \\
Ar-UFB Water & 92.1 \\
Air-UFB Water & 87.1 \\
\hline
\end{tabular}

\section{Skin moisturizing with UFB}


Figure 3 show the results of when the electrical conductivity and the TEWL was measured. Since the permittivity of water is remarkably high as compared with other substance, if the stratum corneum contains a large amount of water, the electrical conductivity becomes large. First, SLS was used as a standard to confirm whether the skin model was successfully prepared. When SLS are dropped onto human skin, it is said that it is impossible to maintain moisture. Usually, the electrical conductivity of human skin is 20 to $30 \mu \mathrm{S}$, but the electrical conductivity of SLS in this experiment was $5.5 \mu \mathrm{S}$, and it was confirmed from the results that water was not retained. Therefore, it can be said that the skin model can be prepared normally. Since $\mathrm{O}_{2}$-UFB water showed the highest electric conductivity of about $80 \mu \mathrm{S}$, which is higher than that of other samples, it is considered that $\mathrm{O}_{2}$-UFB water contain the largest amount of water. It was thought that subdivision of water cluster by bubbling would facilitate permeation and increase water content. However, only the water content of $\mathrm{O}_{2}$-UFB water was high, and the water content of UFB water of other gas species was low. This suggests that oxygen is involved in improving the water content of the stratum corneum of the skin.

TEWL of $\mathrm{O}_{2}$-UFB water showed a lower value than other samples. Further, when compared to the results of the water content, $\mathrm{O}_{2}$-UFB water, since it is possible to remain in the artificial skin compared to other samples, the water content is high, TEWL is considered to be a low result.


Figure 3. Effect on Skin Model (Left: Electrical conductivity, Right: TEWL)

\section{Wettability of UFB to the skin}

Table.3 shows the average contact angle of each sample with respect to each substrate. The contact angle of each sample with respect to the cellophane tape substrate showed almost no difference. On the other hand, it was confirmed that the contact angle of the cellophane tape substrate with the horny layer attached was the smallest for $\mathrm{O}_{2}$ UFB water. Further, the contact angle with UFB water supplied with a gas species other 
than oxygen was not different from that of pure water. From, these results, it is considered that subdivision of water clusters by bubbling does not increase the affinity with the skin, but the higher concentrate of $\mathrm{O}_{2}$-UFB, the higher the affinity with the skin.

As a cause of such a result, it is conceivable that $\mathrm{O}_{2}-\mathrm{UFB}$ is negatively charged. It has been reported that the zeta potential of $\mathrm{O}_{2}$-UFB water used in this study is $-32.4 \mathrm{mV}$. In addition, it has been reported that anionic substance is likely to penetrate the skin ${ }^{6}$. Therefore, it is considered that $\mathrm{O}_{2}$-UFB water with negative zeta potential may easily penetrate into the stratum corneum of the skin and stay there.

TABLE 3. Contact Angle and DO in Each Samples.

\begin{tabular}{cccc}
\hline \multirow{2}{*}{ Sample Name } & \multicolumn{2}{c}{ Contact Angle (degree) } & \multirow{2}{*}{ DO (mg/L) } \\
& Control & Stratum Corneum & \\
\hline Pure Water & 99.0 & 100.1 & 7.90 \\
$\mathrm{O}_{2}$-UFB Water & 97.6 & 85.3 & 26.37 \\
$\mathrm{~N}_{2}$-UFB Water & 98.0 & 106.6 & 1.40 \\
Air-UFB water & 97.9 & 100.9 & 6.78 \\
\hline
\end{tabular}

\section{Conclusion}

In this study, we evaluated the effect of UFB water on human skin. UFB was not irritating to artificial skin in all gas species. In particular, compared to other samples, $\mathrm{O}_{2}$ UFB water had a higher water content in artificial skin and a lower TEWL. From these, it was suggested that $\mathrm{O}_{2}$-UFB water easily penetrates the skin and has the effect of staying in the stratum corneum. In addition, only the contact angle of $\mathrm{O}_{2}$-UFB water was low for the stratum corneum collected by tape stripping. Therefore, it was suggested that $\mathrm{O}_{2}-\mathrm{UFB}$ water has a high affinity with the stratum corneum of the skin.

From these results, $\mathrm{O}_{2}$-UFB water could be expected to be useful for safe and effective cosmetic application.

\section{References}

1. R. Okada, N. Tokunaga, H. Aibara, and Y. Miyakoshi, Japanese Journal of Nursing Art and Science, 9, pp. 45-49 (2010).

2. T. Hirao, Journal of Japanese Cosmetic Science Society, 37, pp. 95-100 (2013). 
3. F. Hashimoto, M. Haruyama, T. Yamashita, and T. Iso, J. Soc. Cosmet. Chem. Japan., p. 23 (1989).

4. T. Uchida, Korean Journal of Chemical Engineering IEEJ Journal of Industry Applications, 5, pp. 1749-1753, (2016).

5. S. Himuro, Progress in multiphase flow research, 2, pp. 39-45 (2001).

6. O. Lee, Skin research and technology, 19 (2012). 